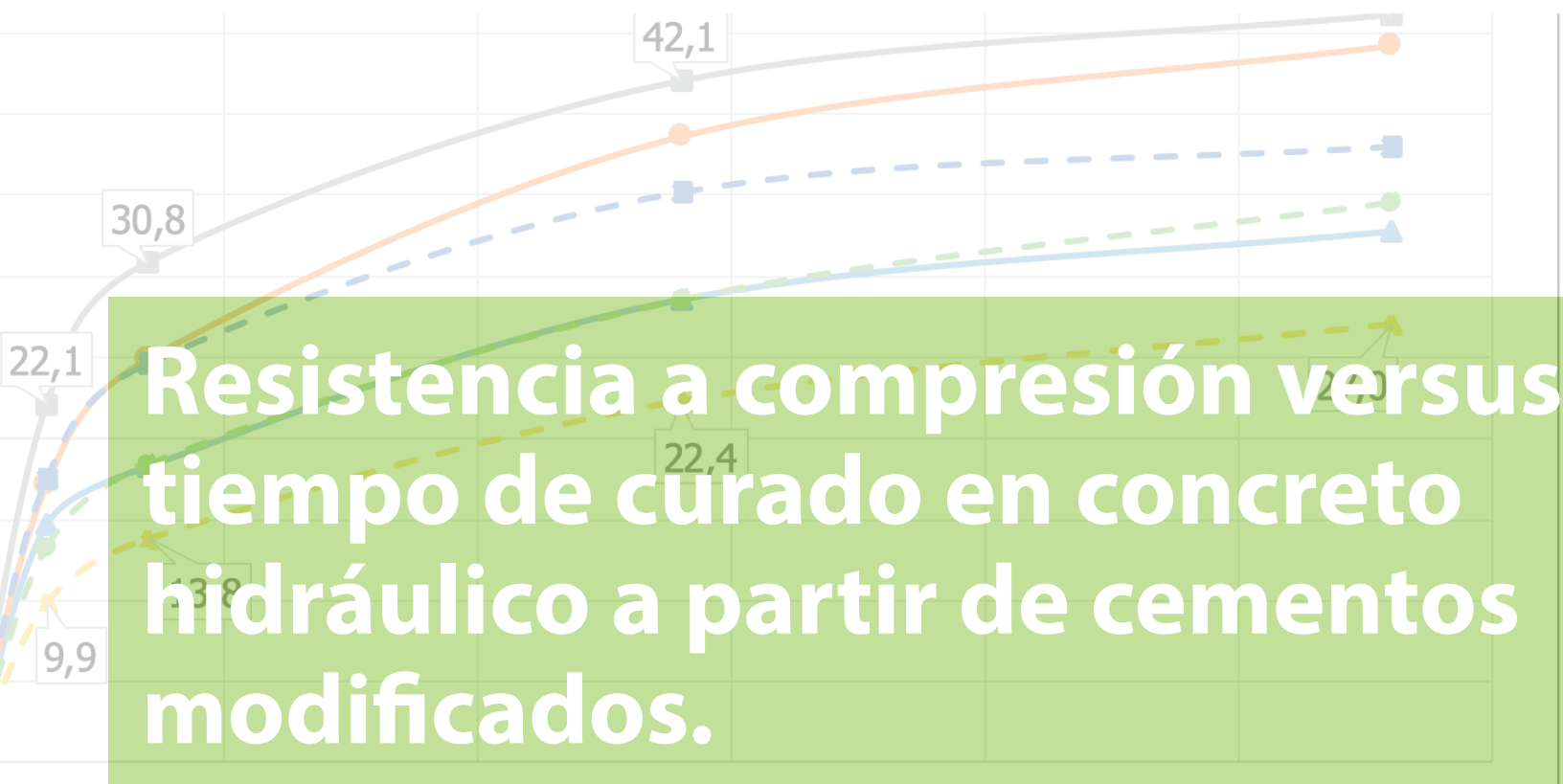
río

cu vuil uyi uyumus y -A2: Cemento MM C ( C -28 con agregados de río

- - A3: Cemento MC/A-AR con agregados de río

A4: Cemento MM C (P. -28 con agregados d tajo

-1-A5: Cemento MC/A-AR con agregados de tajo

Compressive strength versus curing time in hydraulic concrete

from modified cements

Luis Felipe Hernández Mora

Estudiante de Ingeniería Civil

Universidad de Costa Rica, Costa Rica

luisfelipehernandezmora@gmail.com
Ing. Einer Rodríguez Rojas, MAP.

Profesor, Escuela de Ingeniería Civil

Universidad de Costa Rica, Costa Rica

einer.rodríguez@ucr.ac.cr

Ing. Flor de María Muñoz Umaña, M.Sc.

Profesora catedrática, Escuela de Ingeniería Civil

Universidad de Costa Rica, Costa Rica

flor.munoz@ucr.ac.cr

Fecha de recepción: 5 febrero del 2019 / Fecha de aprobación: 20 diciembre del 2019

Índices y Bases de Datos:

latindex ucrindex

\section{- Dialnet DOA}

(2) revistas.ucr.ac.cr/index.php/materiales

(e) lanamme.ucr.ac.cr

@ metodosymateriales.lanamme@ucr.ac.cr
Políticas de Uso:

\section{(C) $\odot \Theta \Theta$}

Revista Métodos y Materiales por LanammeUCR se distribuye bajo: Licencia Creative Commons Atribución-NoComercialSinDerivar 4.0 Internacional. ISSN electrónico: 2215-4558 


\title{
Resistencia a compresión versus tiempo de curado en concreto hidráulico a partir de cementos modificados.
}

\author{
Compressive strength versus curing time in hydraulic concrete \\ from modified cements
}

\author{
Luis Felipe Hernández Mora \\ Estudiante de Ingeniería Civil \\ Universidad de Costa Rica, Costa Rica \\ luisfelipehernandezmora@gmail.com
}

Ing. Flor de María Muñoz Umaña, M.Sc.

Profesora catedrática, Escuela de Ingeniería Civil

Universidad de Costa Rica, Costa Rica

flor.munoz@ucr.ac.cr

\author{
Ing. Einer Rodríguez Rojas, MAP. \\ Profesor, Escuela de Ingeniería Civil \\ Universidad de Costa Rica, Costa Rica \\ einer.rodríguez@ucr.ac.cr
}

\section{RESUMEN}

Este artículo presenta una síntesis de los resultados experimentales obtenidos al ensayar la evolución de la resistencia a la compresión en concretos hechos con distintos cementos modificados, a diferentes edades. Se incluye la caracterización de dos agregados de procedencias distintas (río y tajo) y tres tipos de cemento modificado. Los cementos ensayados corresponden al MM/C(P-C)-28, MM/C(C-P)-28 y MC/A-AR. El objetivo del estudio fue generar las curvas estándar de desarrollo de resistencia de seis mezclas de concreto usando estos cementos y agregados. Las pruebas de caracterización se llevaron a cabo siguiendo los estándares del Instituto de Normas Técnicas de Costa Rica (INTECO) y la Sociedad Americana de Pruebas y Materiales (ASTM). El curado de especímenes de concreto se realizó en cámara húmeda a 4 edades diferentes (3, 7, 28 y 56 días). La resistencia del concreto se obtuvo por medio de ensayos en la Máquina Universal, posteriormente, se procesó y validó estadísticamente los resultados, utilizando los parámetros de aceptación del informe del Instituto Americano del Concreto (ACl 214-2002). A partir del análisis realizado, se logró generar las curvas de desarrollo estándar de resistencia para todos los cementos en estudio, según dos procedencias de agregados e incluyendo un análisis estadístico inferencial para los resultados de resistencia de los concretos. Se concluyó que, al variar la procedencia del agregado para un mismo cemento, su resistencia es significativamente diferente (en términos estadísticos), al igual que para un mismo agregado, cuando se cambia el tipo de cemento.

PALABRAS CLAVE: concreto, resistencia a la compresión, cemento modificado, agregados.

\section{ABSTRACT}

This paper presents a synthesis of the experimental results obtained from testing the evolution of the compressive strength in concrete made with different modified cements at different ages. It includes the characterization of two aggregates of different provenances (river and quarry) and three types of modified cements. The cements tested correspond to MM/C ( $P$ C) $-28, M M / C(C-P)-28$ and MC/A - AR. The objective of the study was to generate the standard resistance development curves of six concrete mixtures (in percentage terms), using these cements and aggregates. The characterization tests were conducted out following the INTECO and ASTM standards. The curing of concrete specimens took place in a humid chamber at 4 different ages (3, 7, 28 and 56 days). The strength of the concrete was obtained through tests on the Universal Machine, and the results were processed and validated statistically, using the acceptance parameters of the ACl 214-2002 report. From the analysis, it was possible to generate the curves of standard development of resistance for all the cements under study using two provenances of aggregates, including an inferential statistical analysis for the resistance results of the concretes. It was concluded that, by varying the origin of the aggregate for the same cement, the resistance of the concrete is significantly different (on statistical terms), as for the same aggregate when the type of cement is changed.

KEY WORDS: concrete, resistance to compression, modified cement, aggregates. 


\section{INTRODUCCIÓN}

La hidratación del cemento es la reacción química que da lugar al endurecimiento del concreto, por lo que maximizar la calidad de este material ha sido uno de los retos de la ingeniería de materiales, desde que inició su utilización masiva, a finales del siglo XIX. Esta investigación se basó en el estudio de la evolución de la resistencia de concretos hechos con cementos modificados, en función del tiempo de curado. Por cemento modificado, se debe entender aquel que resulta de la combinación de clínker tipo I más yeso, y una o varias adiciones minerales que pueden ser caliza, puzolana, escoria de alto horno o humo de sílice.

En Costa Rica, con la aprobación del Reglamento Técnico RTCR 479:2015 Materiales de Construcción, Cementos Hidráulicos $N^{\circ} 39414-M E I C S-S$, cambiaron las especificaciones sobre los tipos de cemento que se comercializaron hasta finales del año 2015. A partir de la aprobación de este Reglamento, surgieron, en el mercado nacional, cementos con adiciones minerales mixtas (de caliza-puzolana).

Hoy día se carece de información relativa al comportamiento de estos cementos cuando se combinan con agregados de diferentes características. A solicitud del cliente, las cementeras y los importadores pueden aportar información sobre el comportamiento del cemento en cuanto a evolución de resistencia, no obstante, más allá de esto, lo que interesa a los constructores de este país es cómo es ese comportamiento cuando se combina con agregados de uso comercial.

La importancia de este proyecto radica en el aporte de conocimiento sobre el desarrollo de la resistencia del concreto cuando se emplea un cemento modificado con agregados nacionales, de características específicas. Considerando que todos los materiales cumplen con especificaciones, incluyendo granulometría en el caso de los agregados, es posible obtener curvas de desarrollo estándar que sirvan para hacer proyecciones de resistencia en el tiempo, a partir de las resistencias obtenidas a edades tempranas.

Se estudió el desarrollo de resistencia a compresión simple en mezclas de concreto hidráulico usando tres tipos de cemento:

- Modificado mixto clase C (puzolana-caliza) - MM/C (P-C)-28

- Modificado mixto clase C (caliza-puzolana) - MM/C (C-P)-28

- Modificado con caliza, de alta resistencia inicial, clase A - MC/A-AR
Los primeros dos cementos tienen una resistencia especificada a 28 días de $28 \mathrm{MPa}$; el tercero, a 1 día de $14 \mathrm{MPa}$ y a 28 días de $38 \mathrm{MPa}$. Las combinaciones de agregado y cemento se detallan en el Cuadro 1.

\begin{tabular}{|c|c|c|c|}
\hline Nomenclatura & Cemento & $\begin{array}{l}\text { Origen del } \\
\text { agregado }\end{array}$ & $\begin{array}{l}\text { Número de } \\
\text { cilindros } \\
\text { ensayados }\end{array}$ \\
\hline $\mathrm{A} 1$ & $\begin{array}{c}\text { CEMENTO } \\
\mathrm{MM} / \mathrm{C}(\mathrm{P}-\mathrm{C})-28\end{array}$ & RÍO & 32 \\
\hline A4 & $\begin{array}{c}\text { CEMENTO } \\
\text { MM/C }(\mathrm{P}-\mathrm{C})-28\end{array}$ & TAJO & 32 \\
\hline $\mathrm{A} 2$ & $\begin{array}{c}\text { CEMENTO } \\
\text { MM/C (C-P) }-28\end{array}$ & RíO & 32 \\
\hline A6 & $\begin{array}{c}\text { CEMENTO } \\
\text { MM/C (C-P) }-28\end{array}$ & TAJO & 32 \\
\hline A3 & $\begin{array}{l}\text { CEMENTO } \\
\text { MC/A - AR }\end{array}$ & RíO & 32 \\
\hline A5 & $\begin{array}{l}\text { CEMENTO } \\
\text { MC/A - AR }\end{array}$ & TAJO & 32 \\
\hline
\end{tabular}

Cada vez que se realizó una mezcla de concreto, se midieron sus características (contenido de aire, temperatura, densidad y asentamiento), con el propósito de ampliar la información del material resultante. El procedimiento se realizó como lo requiere la norma INTE C39:2016, Método de ensayo para la determinación de la resistencia a la compresión uniaxial de especímenes cilindricos de concreto, la cual es equivalente a la norma ASTM C39.

Esta investigación no se planteó variar parámetros como el vibrado, el método de fabricación, la relación de agua/ cemento $(\mathrm{a} / \mathrm{c})$, la calidad del agua o el uso de aditivos.

\section{OBJETIVOS}

General:

Generar curvas de desarrollo de resistencia a compresión simple de concreto hidráulico, a partir de cementos modificados en combinación con agregados de dos procedencias (río y tajo) y que cumplen con las especificaciones de calidad respectivas.

Específicos:

- Caracterizar los agregados y cementos por medio de ensayos de laboratorio, garantizando que cumplen especificaciones estándar y son aptos para la fabricación de concreto hidráulico. 
- Determinar la evolución de la resistencia a 3, 7, 28 y 56 días, mediante pruebas a compresión simple de cilindros de concreto, según tipos de cemento y agregados utilizados.

\section{METODOLOGÍA}

\subsection{Investigación documental}

Se realizó una revisión de estudios relacionados con el tema, con la finalidad de guiar la investigación en formato y metodología. Asimismo, se recopilaron las normas ASTM requeridas para todos los ensayos y sus homólogas normas INTECO.

Con esto se estableció el marco conceptual referente para hacer una caracterización completa de los materiales, seguir un procedimiento en la falla de cilindros, generar un diseño de mezcla, realizar el tratamiento estadístico y observar las tendencias de los resultados experimentales obtenidos.

\subsection{Caracterización de los agregados y cementos}

Cementos

Para esta investigación el cemento se caracterizó mediante los siguientes ensayos de laboratorio:

- INTE C139:2011 Método de ensayo para la determinación deconsistencia normal del cemento hidráulico, equivalente a la norma ASTM C187.

- INTE C137:2012 Método de ensayo para la determinación del tiempo de fragua de un cemento hidráulico por medio de la aguja de Vicat, equivalente a la norma ASTM C191.

- INTE C141:2012 Método de ensayo para la determinación de la densidad del cemento hidráulico, equivalente a la norma ASTM C188.

- INTE C142:2016 Método de ensayo para la determinación de la finura de un cemento hidráulico por medio de la malla de $45 \mu m$ ( $\left.n^{\circ} 325\right)$, equivalente a la norma ASTM C430.

- INTE C57:2008 Método de ensayo para la determinación de la resistencia a la compresión de morteros de cemento hidráulico-Usando especímenes cúbicos de $50 \mathrm{~mm}$, equivalente a la norma ASTM C109.

Con la finalidad de generar valores promedio y obtener mayor validez estadística, se contó con varias réplicas para cada ensayo: 10 para los de consistencia normal y finura del cemento, 7 para el de tiempo de fragua y 8 para el de densidad del cemento hidráulico. Con respecto a los cubos de mortero, se realizaron 12 cubos por cada tipo de cemento y se fallaron en grupos de 3, a las mismas edades de falla que las de los concretos. Esto con la finalidad de contrastar el desarrollo de resistencia del cemento por sí solo (en los cubos de mortero) con el del cemento una vez mezclado con los agregados de interés.

El muestreo del cemento se hizo en concordancia con la norma INTE C153:2015 Extracción de muestras y cantidad de ensayos para cemento hidráulico, equivalente a la norma ASTM C183. Se utilizó un tubo muestreador insertable en una esquina del saco, cruzando hasta el fondo en una dirección preferiblemente diagonal, como lo indica la norma.

\section{Agregados}

Para la caracterización de los agregados, se realizó un ensayo de verificación de granulometría, con el propósito de corroborar que la curva granulométrica se encuentra dentro de los límites especificados según la norma INTE C15:2014, Especificación Normalizada para agregados de concreto, equivalente a la ASTM C33.

Con la finalidad de establecer la desviación estándar de la muestra (a partir del material almacenado y corregido) y una vez definida la granulometría a usar (de donde también se determinó el módulo de finura para ambas fuentes), se realizaron 8 muestreos aleatorios para cada tipo de agregado. Posteriormente, se realizaron 3 réplicas de cada uno de los siguientes ensayos:

- INTE C58:2013 Método de ensayo normalizado para determinar la densidad aparente o peso unitario y porcentaje de vacíos en los agregados, equivalente a la norma ASTM C29.

- INTE C68:2016 Método de ensayo para la densidad, gravedad específica y absorción del agregado grueso, equivalente a la norma ASTM C127.

- INTE C69:2016 Método de ensayo para la densidad, gravedad específica y absorción del agregado fino, equivalente a la norma ASTM C128.

- INTE C59:2015 Método de ensayo normalizado para la detección de impurezas orgánicas en el agregado fino para el concreto, equivalente a la norma ASTM C40.

- INTE C64:2009 Determinación de la resistencia al desgaste de los agregados gruesos, hasta de $37,5 \mathrm{~mm}$, utilizando la máquina de Los Ángeles, equivalente a la norma ASTM C131.

- INTE C29:2016 Determinación de porcentaje de partículas fracturadas, equivalente a la norma ASTM D5821. 
Para llevar a cabo la toma de muestras se siguió la norma INTE C67:2015 Práctica para la toma de muestras de agregados, equivalente a la norma ASTM D75. Se aplicó el procedimiento para muestreo de material cuando este se encuentra apilado.

Para llevar a cabo la reducción de muestras, se siguió la norma INTE C62:2015 Norma para reducir muestras de agregado a tamaño de ensayo, equivalente a la norma ASTM C702. Se usaron separadores mecánicos, que varían según el tamaño de muestra requerida y de si se está reduciendo agregado grueso o fino.

\subsection{Elaboración, curado y falla de especímenes de concreto.}

Siguiendo el procedimiento establecido por la INTE C182016 Fabricación y curado de especímenes en laboratorio, se moldearon los especímenes de concreto. Se ensayaron en la Máquina Universal las 32 muestras de partida en grupos de 8 testigos, a las edades de 3, 7, 28 y 56 días. Como se mencionó anteriormente, se realizaron los ensayos complementarios de caracterización al concreto en estado fresco (asentamiento, densidad, temperatura y contenido de aire). El método de curado fue en cámara húmeda, el cual comenzó 24 horas después de haber sido moldeado el espécimen. El método de coronamiento fue el de pulido y uso de almohadillas de neopreno. El primero se usó en especímenes de edades entre 28 y 56 días, mientras que el segundo, principalmente en especímenes de edades tempranas.

\subsection{Análisis estadístico de los datos}

\section{Estadisticos descriptivos}

El análisis estadístico de los datos se basó en lo propuesto por el informe ACI 214 Evaluación de los resultados de resistencia del concreto. Este documento establece una metodología de control de resultados, donde se analiza la desviación estándar y el coeficiente de variación. En el Cuadro 2, se muestran los valores de desviación estándar para diferentes niveles de control en concretos de $\mathrm{f}^{\prime}{ }_{\mathrm{c}}$ menor a $34.5 \mathrm{MPa}$. Para el caso de concretos con $\mathrm{f}^{\prime}{ }_{\mathrm{c}}$ mayor a 34.5 MPa, el comité del ACI 214 recomienda el uso del coeficiente de variación, pues, para mayores resistencias, este se ve menos afectado (ACI 214, 2009; Cook, 1989). En el Cuadro 3, se muestran los valores de coeficiente de variación para resistencias mayores a los $34.5 \mathrm{MPa}$.

\begin{tabular}{|c|c|c|c|c|c|}
\hline \multicolumn{6}{|c|}{$\begin{array}{l}\text { Cuadro 2. Criterios de aceptación y control para } \\
\text { concreto con } \mathrm{f}_{\mathrm{c}}^{\prime} \text { menor a } 34.5 \mathrm{MPa} \text {. }\end{array}$} \\
\hline \multicolumn{6}{|c|}{$\begin{array}{c}\text { Desviación estándar para diferentes } \\
\text { estándares de control (MPa) }\end{array}$} \\
\hline $\begin{array}{l}\text { Clase de } \\
\text { operación }\end{array}$ & Excelente & $\begin{array}{l}\text { Muy } \\
\text { bueno }\end{array}$ & Bueno & Aceptable & Pobre \\
\hline $\begin{array}{c}\text { Ensayos en } \\
\text { campo }\end{array}$ & Menor a 2.8 & $\begin{array}{c}2.8 \mathrm{a} \\
3.4\end{array}$ & $\begin{array}{c}3.4 \mathrm{a} \\
4.1\end{array}$ & 4.1 a 4.8 & $\begin{array}{c}\text { Mayor } \\
\text { a } 4.8\end{array}$ \\
\hline $\begin{array}{l}\text { Ensayos en } \\
\text { laboratorio }\end{array}$ & Menor a 1.4 & $\begin{array}{c}1.4 \mathrm{a} \\
1.7\end{array}$ & $\begin{array}{c}1.7 \mathrm{a} \\
2.1\end{array}$ & 2.1 a 2.4 & $\begin{array}{c}\text { Mayor } \\
\text { a } 2.4\end{array}$ \\
\hline
\end{tabular}

Fuente: $\mathrm{ACl} 214$

\begin{tabular}{|c|c|c|c|c|c|}
\hline \multicolumn{5}{|c|}{ Puadro 3. Criterios de aceptación y control } \\
Coeficiente de variación para diferentes estándares \\
de control (\%) \\
\hline $\begin{array}{c}\text { Clase de } \\
\text { operación }\end{array}$ & Excelente & $\begin{array}{c}\text { Muy } \\
\text { bueno }\end{array}$ & Bueno & Aceptable & Pobre \\
\hline $\begin{array}{c}\text { Ensayos en } \\
\text { campo }\end{array}$ & Menor a 7 & 7.0 A 9.0 & $\begin{array}{c}9.0 \mathrm{~A} \\
11.0\end{array}$ & $11.0 \mathrm{~A} 14.0$ & $\begin{array}{c}\text { Mayor a } \\
14.0\end{array}$ \\
\hline $\begin{array}{c}\text { Ensayos en } \\
\text { laboratorio }\end{array}$ & $\begin{array}{c}\text { Menor a } \\
3.5\end{array}$ & $3.5 \mathrm{~A} 4.5$ & $\begin{array}{c}4.5 \mathrm{~A} \\
5.5\end{array}$ & $5.5 \mathrm{~A} 7.0$ & $\begin{array}{c}\text { Mayor a } \\
7.0\end{array}$ \\
\hline
\end{tabular}

Fuente: $\mathrm{ACl} 214$

\section{Estadistica inferencial}

Para poder probar la hipótesis de que la resistencia del concreto es diferente al variar la procedencia del agregado y del cemento, se hace necesario el uso de herramientas de estadística inferencial. Las pruebas de análisis de varianza (ANOVA, por sus siglas en inglés) requieren que los datos empleados sigan una distribución normal. Si bien el informe ACI 214 permite la suposición de que los resultados de resistencia del concreto a los 28 días siguen una distribución normal, en este trabajo se probó esta condición a partir de las pruebas de normalidad de Shapiro-Wilk y Jarque-Bera.

Una vez probada la normalidad de los datos, se aplicó una prueba de análisis de varianza de 1 factor, buscando responder la hipótesis nula (si las medias de ambos grupos son distintas). En estos cálculos, la media correspondió a la resistencia a la compresión y los grupos, a concretos hechos con el mismo cemento y diferente tipo de agregado. De igual forma, se probó si la media de los grupos es significativa al variar el tipo de cemento para un mismo tipo de agregado. Finalmente, se realizó una prueba a las 6 muestras, con el propósito de conocer si había al menos 2 que no fueran significativamente diferentes. Esta consistió en una prueba de Tukey, que compara, a través de permutaciones de las muestras, si cada una es significativamente diferente a las demás. Lo anterior 
permitió un análisis de cada caso y amplió la comprensión del efecto de las adiciones minerales en el cemento y, por ende, en la mezcla, cuando varía la procedencia del agregado.

La resistencia analizada en la prueba de análisis de varianza es la correspondiente a 28 días de edad, pues los resultados de los Cuadros 2 y 3 son generalizables solo para esta edad (ACI 214).

\section{RESULTADOS}

\subsection{Caracterización de los agregados y cementos}

Como parte del aseguramiento de que los materiales usados cumplen con especificaciones estándar de calidad, se realizó una caracterización de los agregados y cementos. En el Cuadro 4, se muestran los resultados experimentales de la caracterización de los agregados finos ensayados, de procedencias tanto de río como de tajo.

Para el ensayo de granulometría, se realizaron 8 réplicas con el fin de obtener la desviación estándar entre muestras, mientras que, para los demás ensayos de caracterización, se hicieron 3. En el Cuadro 5, se muestran los resultados experimentales de la caracterización de los agregados gruesos, tanto de río como de tajo.

En el Cuadro 6, se muestran los resultados experimentales de la caracterización de los cementos.

\begin{tabular}{|c|c|c|c|c|c|c|c|c|c|}
\hline \multicolumn{10}{|c|}{ Propiedad física } \\
\hline Origen & $\begin{array}{l}\text { Módulo de } \\
\text { Finura }\end{array}$ & $\begin{array}{c}\text { Gravedad } \\
\text { específica seca }\end{array}$ & $\begin{array}{l}\text { Gravedad } \\
\text { específica } \\
\text { SSS }\end{array}$ & D (\%) & $\begin{array}{l}\delta \text { suelto } \\
\left(\mathrm{kg} / \mathrm{m}^{3}\right)\end{array}$ & $\begin{array}{c}\delta \text { varillado } \\
\left(\mathrm{kg} / \mathrm{m}^{3}\right)\end{array}$ & $W_{\text {lab }}(\%)$ & $\begin{array}{l}\text { Relación de } \\
\text { vacios (\%) }\end{array}$ & $\begin{array}{l}\text { Impurezas } \\
\text { orgánicas }\end{array}$ \\
\hline Río & 3.02 & 2.49 & 2.59 & 4.0 & 1544 & 1640 & 1.7 & 79 & Conforme \\
\hline Tajo & 2.74 & 2.55 & 2.60 & 2.1 & 1264 & 1523 & 2.7 & 77 & Conforme \\
\hline $\begin{array}{l}\text { SSS: En cor } \\
\text { D: Absorcic } \\
\delta: \text { Peso un }\end{array}$ & $\begin{array}{l}\text { lición saturada } \\
\text {, porcentaje en } \\
\text { ario total }\end{array}$ & $\begin{array}{l}\text { uperficie seca } \\
\text { masa }\end{array}$ & & & & & & & \\
\hline
\end{tabular}

\begin{tabular}{|c|c|c|c|c|c|c|c|c|c|c|}
\hline \multicolumn{11}{|c|}{ Propiedad física } \\
\hline Origen & $\begin{array}{c}\text { Tamaño real } \\
(\mathrm{mm})\end{array}$ & $\begin{array}{c}\text { Gravedad } \\
\text { específica seca }\end{array}$ & $\mid \begin{array}{c}\text { Gravedad } \\
\text { específica SSS }\end{array}$ & D (\%) & $\begin{array}{l}\delta \text { suelto } \\
\left(\mathrm{kg} / \mathrm{m}^{3}\right)\end{array}$ & $\begin{array}{c}\delta \text { varillado } \\
\left(\mathrm{kg} / \mathrm{m}^{3}\right)\end{array}$ & $\begin{array}{l}W_{\text {lab }} \\
(\%)\end{array}$ & $\begin{array}{l}\text { Relación de } \\
\text { vacíos (\%) }\end{array}$ & R.A. (\%) & P.F. (\%) \\
\hline Río & 16 & 2.39 & 2.44 & 1.8 & 1480 & 1578 & 1.4 & 68 & 85 & 99 \\
\hline Tajo & 12.5 & 2.56 & 2.60 & 1.4 & 1489 & 1557 & 1.1 & 78 & 81 & 100 \\
\hline \multicolumn{11}{|c|}{$\begin{array}{l}\text { D: Absorción, porcentaje en masa } \\
\text { d: Peso unitario total } \\
\text { SSS: En condición saturada superficie seca } \\
\text { R.A: Resistencia a la abrasión, porcentaje recuperado } \\
\text { P.F: Partículas fracturadas }\end{array}$} \\
\hline
\end{tabular}

\begin{tabular}{|c|c|c|c|c|c|c|c|c|c|c|}
\hline \multirow{3}{*}{$\begin{array}{l}\text { Tipo de } \\
\text { cemento }\end{array}$} & \multicolumn{10}{|c|}{ Propiedad física } \\
\hline & \multirow{2}{*}{$\begin{array}{c}\text { Consistencia normal } \\
(\mathrm{a} / \mathrm{c})(\%)\end{array}$} & \multirow{2}{*}{$\begin{array}{l}\text { Densidad ( } \mathrm{g} / \\
\mathrm{cm} 3)\end{array}$} & \multicolumn{2}{|c|}{$\begin{array}{l}\text { Tiempo } \\
\text { de fragua } \\
\text { (minutos) }\end{array}$} & \multirow{2}{*}{$\begin{array}{c}\text { Finura } \\
(\%)\end{array}$} & \multicolumn{4}{|c|}{ Resistencia a compresión (MPa) } & \multirow[t]{2}{*}{$\mathrm{pH}$} \\
\hline & & & Inicial & Final & & 3 días & 7 días & 28 días & 56 días & \\
\hline $\begin{array}{c}\mathrm{MM} / \mathrm{C} \\
(\mathrm{P}-\mathrm{C})-28\end{array}$ & 28.0 & 2.81 & 136 & 245 & 93.7 & 13.8 & 18.7 & 27.5 & 31.4 & 11.64 \\
\hline $\begin{array}{c}\mathrm{MM} / \mathrm{C} \\
(\mathrm{C}-\mathrm{P})-28\end{array}$ & 28.0 & 2.84 & 110 & 200 & 95.8 & 16.7 & 20.5 & 29.9 & 34.2 & 11.02 \\
\hline MC/A-AR & 28.0 & 3.10 & 141 & 275 & 96.5 & 21.4 & 30.3 & 36.6 & 39.3 & 11.73 \\
\hline
\end{tabular}




\subsection{Moldeo y ensayo de especímenes de concreto}

Siguiendo el procedimiento descrito en INTE-C39:2016, se obtuvo la resistencia de los concretos ensayados. Con la finalidad de obtener la tasa de desarrollo de resistencia del concreto, esta se expresó en términos porcentuales, tomando la resistencia a los 28 días como la resistencia al $100 \%$. En la Figura 1, se muestran los resultados de los concretos hechos con cemento MM/C (P-C)-28. Con el fin de aislar la influencia de los agregados, se muestra también la resistencia porcentual obtenida por cubos de mortero, a esas mismas edades.

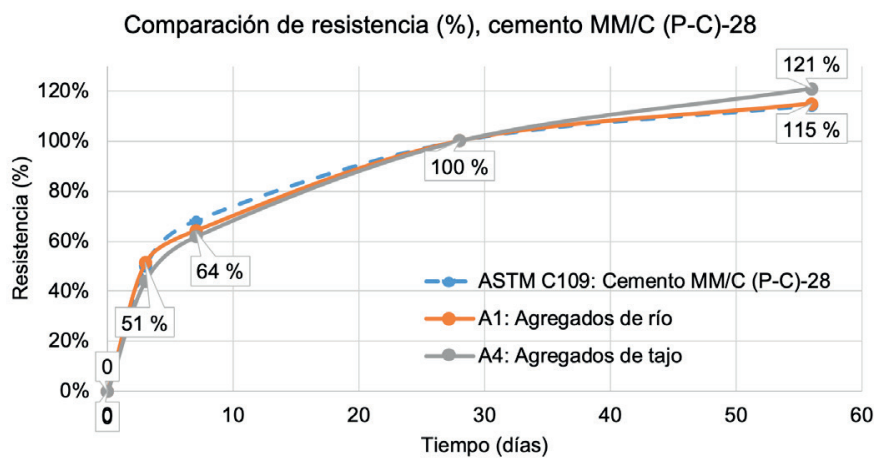

Figura 1. Comparación de resistencia entre concretos A1, A4 y cubos de mortero hechos con MM/C (P-C)-28.

En las Figuras 2 y 3, se muestran los resultados de concretos hechos con los cementos MM/C (C-P)-28 y MC/A-AR, respectivamente.

Comparación de resistencia (\%), cemento MM/C (C-P)-28

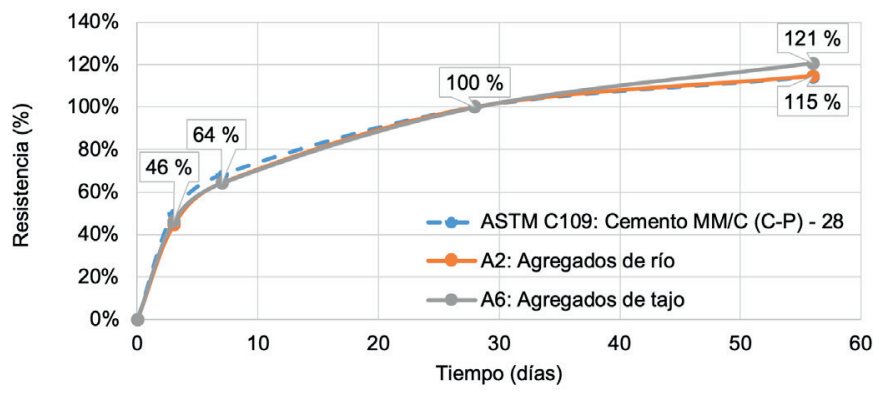

Figura 2. Comparación porcentual de resistencia entre concretos A2, A6 y cubos de mortero hechos con MM/C (C-P)-28.

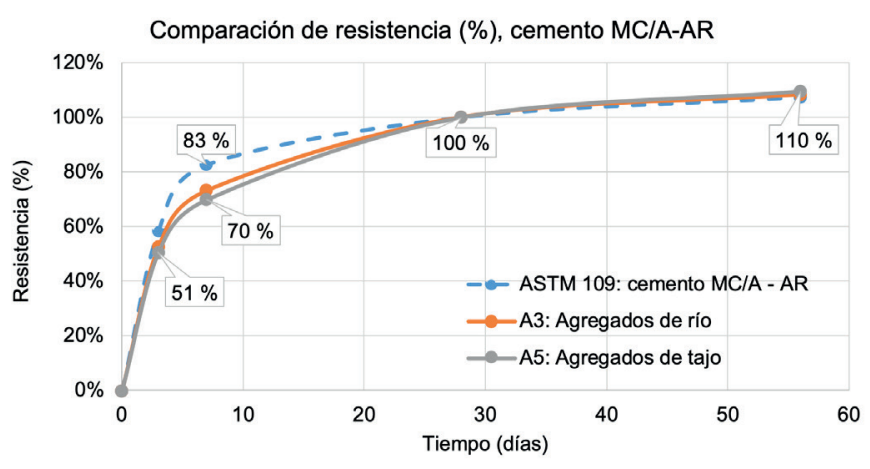

Figura 3. Comparación porcentual de resistencia entre concretos $A 3$, A5 y cubos de mortero hechos con MC/A-AR.

\subsection{Análisis estadístico de los datos}

En el Cuadro 7, se muestran los estadísticos descriptivos de la resistencia a la compresión a los 28 días de edad, para las 6 mezclas de concreto ensayadas. Como se mencionó, se usa la resistencia a los 28 días, pues los resultados del Informe ACI 214 son válidos únicamente para esta edad. En el caso de que la resistencia fuera mayor a $34.5 \mathrm{MPa}$, se usó el coeficiente de variación, en lugar de la desviación estándar. La clasificación de la calidad estadística de los resultados fue dada a partir del valor de la desviación estándar o del coeficiente de variación, según aplicara. Para garantizar una mayor precisión, en algunos casos se eliminaron datos: el mayor o el menor (según cuál afectaba más la desviación estándar).

\begin{tabular}{|c|c|c|c|c|c|c|}
\hline \multicolumn{7}{|c|}{ Cuadro 7. Calidad estadística de los resultados, según estadísticos descriptivos. } \\
\hline Mezcla de concreto & Concreto A1 & Concreto A2 & Concreto A3 & Concreto A4 & Concreto A5 & Concreto A6 \\
\hline Edad /Parámetro estadístico & 28 días & 28 días & 28 días & 28 días & 28 días & 28 días \\
\hline Resistencia promedio & 28.5 & 38.6 & 42.1 & 22.4 & 35.2 & 28.5 \\
\hline Desviación estándar & 1.5 & 2.1 & 1.8 & 1.0 & 1.7 & 0.7 \\
\hline Coeficiente de variación (\%) & 5.4 & 5.6 & 4.4 & 4.4 & 4.9 & 2.6 \\
\hline Especímenes tomados en cuenta & 6 & 6 & 8 & 8 & 7 & 8 \\
\hline Clasificación según ACl 214 & Muy bueno & Aceptable & Muy bueno & Excelente & Bueno & Excelente \\
\hline
\end{tabular}


Cabe destacar que si bien se eliminaron algunos datos para obtener un promedio de resistencia más certero y, así, una calidad estadística mayor, esto fue únicamente para la generación de las curvas de resistencia. Esto no fue así para las pruebas de análisis de varianza Anova, que exigen muestras del mismo tamaño. El Cuadro 8 ofrece los resultados de la prueba de Tukey (en la que se compararon las 6 mezclas de concreto) y permite responder la siguiente pregunta: ¿Existe una diferencia significativa entre las resistencias de cada una de estas mezclas de concreto, si se las compara una a una? la procedencia del agregado (esto se determina al observar que una curva se sitúa sobre la otra). Asimismo, es interesante destacar que, en todos los casos, el concreto se comportó de una forma muy similar a la pasta de mortero. Esto indica que el desarrollo de resistencia (en términos porcentuales) no depende de la procedencia del agregado, sino más bien del tipo de cemento. Comparando la resistencia obtenida a los 7 días por los concretos hechos con cemento MM/C (P-C)28 y MM/C (C-P)-28, respectivamente, queda en evidencia que el desarrollo de resistencia es prácticamente el mismo. Este comportamiento sugiere que, en realidad, la rapidez

\begin{tabular}{|c|c|c|c|c|c|}
\hline Contraste & Diferencia & Diferencia estandarizada & Valor crítico & Pr $>$ Diff & Significancia \\
\hline $\mathrm{A} 3$ vs $\mathrm{A} 4$ & 19.726 & 19.737 & 2.985 & $<0.0001$ & Sí \\
\hline$A 3$ vs $A 6$ & 13.562 & 13.569 & 2.985 & $<0.0001$ & Sí \\
\hline $\mathrm{A} 3$ vs $\mathrm{A} 1$ & 13.488 & 13.495 & 2.985 & $<0.0001$ & Sí \\
\hline $\mathrm{A} 3$ vs $\mathrm{A} 5$ & 7.458 & 7.462 & 2.985 & $<0.0001$ & Sí \\
\hline $\mathrm{A} 3$ vs $\mathrm{A} 2$ & 3.521 & 3.523 & 2.985 & 0.012 & Sí \\
\hline $\mathrm{A} 2$ vs $\mathrm{A} 4$ & 16.205 & 16.214 & 2.985 & $<0.0001$ & Sí \\
\hline$A 2$ vs $A 6$ & 10.041 & 10.046 & 2.985 & $<0.0001$ & Sí \\
\hline $\mathrm{A} 2$ vs $\mathrm{A} 1$ & 9.967 & 9.972 & 2.985 & $<0.0001$ & Sí \\
\hline A2 vs A5 & 3.937 & 3.939 & 2.985 & 0.004 & Sí \\
\hline A5 vs A4 & 12.268 & 12.275 & 2.985 & $<0.0001$ & Sí \\
\hline A5 vs $A 6$ & 6.104 & 6.107 & 2.985 & $<0.0001$ & Sí \\
\hline $\mathrm{A} 5$ vs $\mathrm{A} 1$ & 6.030 & 6.033 & 2.985 & $<0.0001$ & Sí \\
\hline $\mathrm{A} 1$ vs $\mathrm{A} 4$ & 6.238 & 6.241 & 2.985 & $<0.0001$ & Sí \\
\hline$A 1$ vs $A 6$ & 0.074 & 0.074 & 2.985 & 1.000 & No \\
\hline A6 vs $A 4$ & 6.164 & 6.167 & 2.985 & $<0.0001$ & Sí \\
\hline
\end{tabular}

Como se observa, solamente en el caso de las mezclas A1 y A6 no existe una diferencia en el resultado de la resistencia, al variar la procedencia del agregado y el tipo de cemento.

\section{DISCUSIÓN}

En el Cuadro 6, se puede observar que la resistencia a los 28 días de los cementos mixtos modificados clase $\mathrm{C}$ (MM/C) llega a los $28 \mathrm{MPa}$, mediante la prueba de resistencia de cubos de mortero. Asimismo, los tiempos de fragua (iniciales y finales) cumplen con lo establecido en la norma INTE C147-2015.

Las pendientes de las gráficas en las Figuras 1,2 y 3 permiten apreciar que, en general para ambos tipos de concreto, el desarrollo porcentual de la resistencia es muy similar al variar con la que el concreto desarrolla su resistencia depende del porcentaje de clínker y no tanto de las adiciones minerales con el que está mezclado (en el caso de cementos modificados).

Por otra parte, cuando el porcentaje de resistencia obtenido se contrasta con el del concreto hecho en MC/A-AR, sí se observa una diferencia sustancial (de hasta $20 \%$ en cubos de mortero) en la forma en que se obtiene la resistencia.

En las Figuras 1, 2 y 3, se aprecia cómo, en las edades iniciales, la pasta de mortero presenta un desarrollo levemente más rápido de la resistencia, sin embargo, en edades avanzadas, esta resistencia es compensada por los concretos (independientemente de la adición mineral). Lo anterior puede atribuirse a que la puzolana favorece un desarrollo más lento de la resistencia. 


\section{Porcentaje de diferencia entre las mezclas}

Según se planteó en la hipótesis, los porcentajes de proyección de la resistencia no difieren entre una mezcla de concreto y otra (variando el tipo de agregado) en más de un $10 \%$. En esta investigación, se obtuvo que la mayor variación sucede en edades avanzadas, donde los concretos desarrollan resistencia hasta con un $7 \%$ de diferencia.

Diferencia estadísticamente significativa de la resistencia al variar el agregado y el cemento

En la sección de resultados, el Cuadro 8 hace referencia a la prueba Anova, aplicada a todas las mezclas de concreto. Con estas pruebas (y posteriormente la de Tukey), donde cada cemento varía el tipo de agregado (3 pruebas) y cada agregado varía el tipo de cemento ( 2 pruebas), se obtuvo, en todos los casos, que la resistencia del concreto a los 28 días es significativamente diferente entre dos mezclas que presenten una de estas variantes.

\section{CONCLUSIONES}

1. Los agregados finos y gruesos utilizados en el proyecto cumplen con los límites granulométricos establecidos en la INTE C15:2014 (ASTM C33).

2. En todos los casos, el tiempo de fragua y la resistencia a compresión en cubos de mortero de los cementos cumplen con los valores establecidos en la norma INTE C147:2015.

3. Bajo un curado adecuado, el concreto desarrolla, en la primera semana, al menos el $65 \%$ de la resistencia proyectada a 28 días.

4. Cuando se usan adiciones minerales de caliza y puzolana en los cementos (MM/C (P-C)-28 y MM/C (C-P)-28), disminuyen la densidad, la finura y el tiempo de fragua con respecto al cemento sin adiciones (MC/A-AR).

5. La desviación estándar y el coeficiente de variación califican los resultados de resistencia obtenidos, según el criterio del ACI 214, como: excelentes, muy buenos, buenos y, solo en un caso (concreto A2), aceptables. Por lo anterior, se concluye que los datos de resistencia del concreto son válidos y aplicables. Eso respalda, además, que el método de moldeo y ensayo fue el adecuado.

6. La preponderancia de caliza en la mezcla (C-P) con respecto a la $(\mathrm{P}-\mathrm{C})$ genera una fragua más rápida y una mayor resistencia en el concreto.
7. En todos los concretos hechos con agregados de río, se obtuvo resistencias mayores a las de los concretos hechos con el mismo tipo de cemento y agregados de tajo.

8. Para un mismo tipo de agregado, todos los cementos generan concretos de diferente resistencia.

9. Los cementos mixtos modificados ganan gran parte de su resistencia en edades avanzadas, así, a los 56 días, llegan a obtener hasta un $21 \%$ adicional a la resistencia obtenida a los 28 días.

10. La resistencia porcentual de concretos hechos con un mismo cemento, pero diferente agregado, difiere mayormente a edades tempranas. A edades avanzadas, ese comportamiento se estabiliza y oscila entre $5 \%$ y $7 \%$, como se planteó en la hipótesis.

11. Al compararse con el desarrollo porcentual de los cubos de mortero, tanto el agregado de río como el de tajo retrasan la obtención de resistencia en una edad temprana (en términos porcentuales). Sin embargo, el concreto compensa esa ganancia de resistencia en edades más avanzadas (después de los 28 días).

12. Para cada tipo de cemento existe una diferencia significativa en la resistencia obtenida, según se use agregado de río o de tajo.

\section{AGRADECIMIENTOS}

Los autores agradecen al LanammeUCR, por facilitar las instalaciones y el equipo, todo debidamente calibrado, para la ejecución de los ensayos. 


\section{ANEXOS}

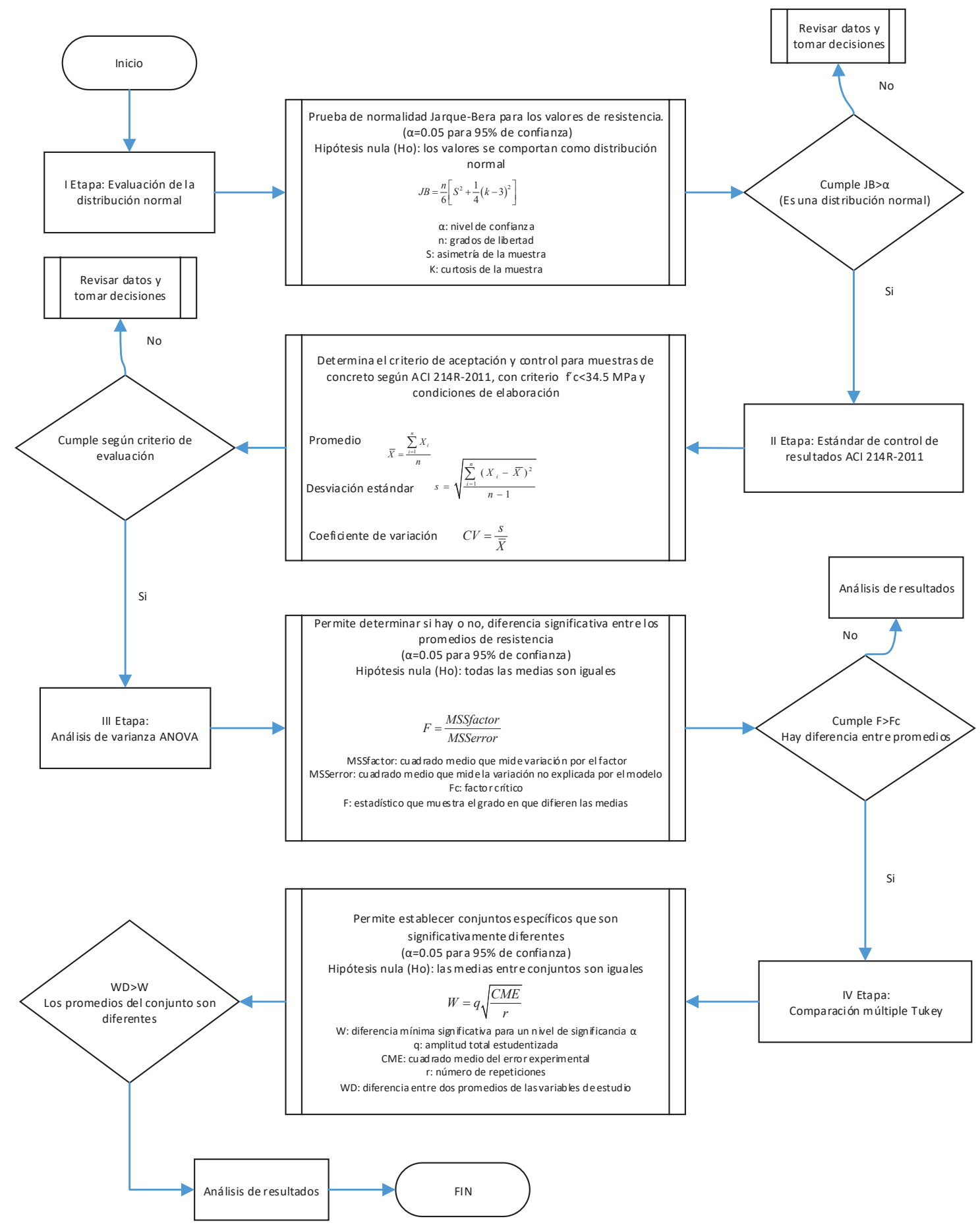

Diagrama de flujo estadística 


\section{REFERENCIAS}

Decreto Ejecutivo Nº 39414-MEIC-S Reglamento Técnico RTCR 479:2015 Materiales de Construcción, Cementos Hidráulicos.

Instituto Americano del Concreto. (2011). ACI 214R-11R-2011. Evaluación de los resultados de resistencia del concreto. American Concrete Institute: Michigan, Estados Unidos de América.

Instituto de Normas Técnicas de Costa Rica. (2018). INTE C15:2018. Agregados para concreto - Requisitos (MOD). Correspondencia: ASTM C33/C33M-18.

- Instituto de Normas Técnicas de Costa Rica (2016). INTE C18:2016. Práctica normalizada para preparación y curado de especímenes de concreto para ensayo en laboratorio. Correspondencia: ASTM C192/C192M-16a.

- Instituto de Normas Técnicas de Costa Rica. (2017). INTE C22:2017. Uso de almohadillas no adheridas en la determinación del esfuerzo de compresión de cilindros de concreto endurecido. Correspondencia: ASTM C1231/C1231M-15.

Instituto de Normas Técnicas de Costa Rica. (2016). INTE C29:2016. Método de prueba para determinar el porcentaje de partículas fracturadas en el agregado. Correspondencia: ASTM D5821-13.

- Instituto de Normas Técnicas de Costa Rica. (2018). INTE C39:2018.Resistencia a la compresión uniaxial de especímenes cilíndricos de concreto. Método de ensayo. Correspondencia: ASTM C39/C39M-18.

- Instituto de Normas Técnicas de Costa Rica. (2008). INTE C57:2008. Método de ensayo para la resistencia a la compresión de morteros de cemento hidráulico - Usando especímenes cúbicos de 50 mm. Correspondencia: ASTM C109M-05.

- Instituto de Normas Técnicas de Costa Rica. (2013). INTE C58:2013. Método de ensayo normalizado para determinar la densidad aparente o peso unitario y porcentaje de vacíos en los agregados. Correspondencia: ASTM C29-09.

Instituto de Normas Técnicas de Costa Rica. (2015). INTE C59:2015. Determinación de las impurezas en el agregado fino. Correspondencia: ASTM C40/C40M-11.

- Instituto de Normas Técnicas de Costa Rica. (2015). INTE C62:2015. Norma para la reducir muestras de agregado a tamaño de ensayo. Correspondencia: ASTM C702-98(03)/ C702M-11.

- Instituto de Normas Técnicas de Costa Rica. (2017). INTE C64:2017. Determinación de la resistencia al desgaste de los agregados gruesos hasta de $37,5 \mathrm{~mm}$, utilizando la máquina De Los Ángeles. Correspondencia: ASTM C131-14.
- Instituto de Normas Técnicas de Costa Rica. (2015). INTE C67:2015. Práctica para la toma de muestras de agregados. Correspondencia: ASTM D75/D75M-14.

- Instituto de Normas Técnicas de Costa Rica. (2016). INTE C68:2016. Determinación de la densidad relativa (gravedad específica) y la absorción del agregado grueso. Método de ensayo. Correspondencia: ASTM C127-15.

Instituto de Normas Técnicas de Costa Rica. (2016). INTE C69:2016. Determinación de la densidad relativa (gravedad específica) y la absorción del agregado fino. Método de ensayo. Correspondencia: ASTM C128-15.

- Instituto de Normas Técnicas de Costa Rica. (2012). INTE C137:2012. Determinación del tiempo de fragua por aguja de Vicat. Método de ensayo. Correspondencia: ASTM C191-08.

Instituto de Normas Técnicas de Costa Rica. (2018). INTE C139:2018. Método de ensayo para la determinación de la consistencia normal del cemento hidráulico. Correspondencia: ASTM C187 - 16.

Instituto de Normas Técnicas de Costa Rica. (2018). INTE C141:2018. Método de ensayo para la determinación de la densidad del cemento hidráulico. Correspondencia: ASTM C188-17.

Instituto de Normas Técnicas de Costa Rica. (2018). INTE C142:2018. Determinación de la finura de un cemento hidráulico por medio de la malla de $45 \mathrm{~mm}\left(\mathrm{~N}^{\circ} 325\right)$. Método de ensayo. Correspondencia: ASTM C430-17.

Instituto de Normas Técnicas de Costa Rica. (2015). INTE C147:2018. Cemento hidráulico - Requisitos. Correspondencia: No aplica.

- Instituto de Normas Técnicas de Costa Rica. (2018). INTE C153:2018. Muestreo y cantidad de ensayos de cemento hidráulico. Correspondencia: ASTM C183-16. 